\title{
Resurgimiento de enfermedad hemorrágica del recién nacido. Implicaciones para su prevención
}

\author{
O scar Alberto N ewton-Sánchez, M en C, ${ }^{(1)}$ Gloria Basurto-Celaya, Biól, ${ }^{(1)}$
}

Vesta Richardson, MC, ${ }^{(1)}$ Jaime Belkind-Gerson, MC.(1)

\section{Newton-Sánchez OA, Basurto-Celaya G, Richardson V, Belkind-Gerson J. Resurgimiento de enfermedad hemorrágica del recién nacido. Implicaciones para su prevención. Salud Publica Mex 2002;44:57-59.}

El texto completo en inglés de este artículo está disponible en: http://www.insp.mx/salud/index.html

\section{Resumen}

Objetivo. Describir la experiencia con enfermedad hemorrágica del recién nacido en un hospital pediátrico del tercer nivel de atención. Material y métodos Estudio retrospectivo efectuado en el Hospital del $N$ iño Morelense de la ciudad de Cuernavaca, estado de Morelos, México, durante el periodo 1997-2000, con una muestra de 46 neonatos, afectados de enfermedad hemorrágica del recién nacido (EHRN), referidos de poblaciones de la periferia del estado. Resultados De los pacientes, $91 \%$ presentó la forma tardía grave de EHRN . El $52 \%$ de los partos fueron atendidos por médico y $48 \%$ por parteras. Se desconoce aplicación de vitamina $\mathrm{K}$ en $61 \%$, en $35 \%$ no fue aplicada y en $4 \%$ sí se aplicó. La mayoría presentó sintomatología severa secundaria a hemorragia intracraneana. Hubo defunción en $11 \%$ y secuelas severas en $41 \%$. Conclusiones. La EHRN es un problema frecuente en el estado de Morelos y, por ello, se requiere analizar los programas de salud reproductiva e intensificar la capacitación de médicos y enfermeras sobre la prevención de ésta mediante el uso de vitamina K.El texto completo en inglés de este artículo está disponible en: http://www.insp.mx/salud/index.html

Palabras clave: enfermedad hemorrágica del recién nacido; vitamina K; México

\author{
Newton-Sánchez OA, Basurto-Celaya G, \\ Richardson V, Belkind-Gerson J. \\ Is the hemorrhagic disease \\ of the newborn re-emerging? \\ Issues related to its prevention. \\ Salud Publica Mex 2002;44:57-59. \\ The English version of this paper \\ is available at: http://www.insp.mx/salud/index.html
}

\begin{abstract}
Objective.To describe the occurrence of hemorrhagic disease of the newborn (HDN) at a tertiary care pediatric hospital of Morelos state. Material and Methods A retrospective case series study was conducted between 19972000 at Hospital del Niño Morelense (Morelos State Children's Ho spital), in 46 newborns aged under 12 weeks. Study subjects were referred from peripheral units with a diagnosis of HDN . Results. The severe late-onset form of HDN was present in $91 \%$ of the cases. Fifty-two percent of childbirths were assisted by a physician and $48 \%$ by an empiric midwife. Application of vitamin $\mathrm{K}$ was unknown in $61 \%$ of cases, in 39\% it was not applied and in 4\% it was applied. The majority of infants presented severe symptoms due to intra-cranial bleeding, $11 \%$ died, and $41 \%$ had severe disease sequelae. Conclusions Given the high prevalence of HDN in the State of Morelos, reproductive health programs should be reviewed and training programs intensified to promote the utilization of vitamin $\mathrm{K}$ by physicians and nurses for preventing this disease. The English version of this paper is available at: http://www.insp.mx/salud/ index.html
\end{abstract}

Key words: hemorrhagic disease of newborn; vitamin K; Mexico

(1) Servicio de Enseñanza e Investigación, Hospital del N iño Morelense, Cuernavaca, Morelos, México.

Fecha de recibido: 2 de abril de 2001 - Fecha de aprobado: 28 de agosto de 2001

Solicitud de sobretiros: Jaime Belkind-Gerson. Jefatura de Investigación. Hospital del N iño Morelense. G ustavo Gómez Azcárate 205, Lomas de la Selva, 62270 Cuernavaca, Morelos, México.

Correo electrónico: cuernavacajaime@ yahoo.com 
L enfermedad hemorrágica clásica del recién nacido descrita en 1894 se manifiesta en la primera semana de vida con sangrado en piel o tubo digestivo; ${ }^{1}$ la enfermedad de presentación tardía, descrita en 1967, es un síndrome grave, manifestado en casi la mitad de los casos por hemorragia cerebral súbita en las primeras 12 semanas de edad. ${ }^{2}$ Ambas presentaciones pueden ser clínicamente severas, causando daño cerebral e incluso la muerte, lo cual se puede evitar con la administración profiláctica de vitamina $\mathrm{K}$ al nacimiento, como parte del manejo de todo recién nacido. ${ }^{3}$

La deficiencia severa de vitamina $\mathrm{K}$ se desarrolla con mayor frecuencia en los niños y niñas alimentados exclusivamente en el seno materno, ya que el aporte de vitamina $\mathrm{K}$ es casi nulo, ${ }^{2-4}$ y la concentración en sangre de factores de la coagulación dependientes de vitamina $\mathrm{K}$, en recién nacidos a término, representa aproximadamente $50 \%$ de la de adultos normales. Además del deficiente aporte de vitamina $\mathrm{K}$ en la leche materna, otros factores de riesgo son diarrea crónica, función hepática alterada con colestasis, disminución de la absorción e intoxicación o infección hepática. ${ }^{3}$ Este problema puede prevenirse casi en su totalidad al elevar los niveles de protrombina con la administración oral o parenteral de vitamina $\mathrm{K}$ al nacimiento. ${ }^{3-5}$

El objetivo de este trabajo es reportar la experiencia de cuatro años en el manejo de niños con EHRN, referidos de unidades periféricas del estado de Morelos, en un hospital pediátrico de tercer nivel de atención.

\section{Material y métodos}

Se hizó revisión de los expedientes de casos de EHRN atendidos en el Hospital del Niño Morelense en Cuernavaca, Morelos, México, durante el periodo 1997-2000, verificando que los diagnósticos de EHRN se hubiesen establecido de acuerdo con la Clasificación Internacional de Enfermedades. ${ }^{6}$ Todos los diagnósticos se basaron en 1) edad, igual o menor a 12 semanas de vida, 2) hemorragia interna o externa, 3) tiempos de coagulación iguales o mayores al doble del normal, 4) cultivos negativos y sin signos clínicos y de laboratorio de infección sistémica, 5) plaquetas normales, 6) no colestasis y 7) normalización de tiempos de coagulación con la aplicación parenteral de vitamina $\mathrm{K}$.

\section{Resultados}

Se revisaron 46 expedientes de pacientes con diagnóstico confirmado de EHRN. El 63\% (29) fueron del sexo masculino. El promedio de edad al momento del diagnóstico fue de 33.5 días, con un rango de 2 a 60 . Todos tenían al nacimiento más de 38 semanas de edad gesta- cional. El 89\% (41) recibió alimentación exclusiva en el seno materno. La presentación clásica o temprana de EHRN se manifestó en 9\% (4) y la tardía en 91\% (42). El $52 \%$ (24) fueron atendidos por médico y el resto por parteras empíricas. En 61\% (28) la madre desconocía el antecedente de aplicación de vitamina K, en 35\% (16) no fue aplicada y únicamente en $4 \%$ (2) se aplicó la profilaxis. La presentación clínica al ingreso fue irritabilidad en $87 \%$, palidez en $76 \%$, fontanela anterior abombada en $72 \%$, hemorragia intracraneana en $67 \%$, vómitos en $61 \%$, crisis convulsivas en $46 \%$ (cuadro I). Respecto a las complicaciones, hubo secuelas neurológicas severas en $41 \%$ (19), moderadas en $2 \%$ (1), leves en $9 \%$ (4) y en 37\% (17) no las hubo; $11 \%$ (5) presentó defunción, secundaria a la hemorragia y a la hipertensión intracraneanas.

\section{Discusión}

La EHRN es un problema con consecuencias graves o fatales en el estado de Morelos si tomamos en cuenta un promedio por año de 15000 nacidos vivos en Morelos y lo relacionamos con los casos de EHRN, vistos únicamente en nuestro hospital, la incidencia de este padecimiento sería de aproximadamente 80 por 100 000, la cual, hasta la fecha, es la más elevada informada mundialmente, por lo cual representa un problema de salud pública estatal.

\section{Cuadro I \\ Manifestaciones CLÍNicas de enfermedad HEMORRÁGICA DEL RECIÉN NACIDO EN EL HOSPITAL DEL NiÑo MORELENSE. MÉXICO, 1997-2000}

\begin{tabular}{lll}
\multicolumn{1}{l}{ Signos y síntomas } & Número & $\%$ \\
Irritabilidad & 40 & $86.9 \%$ \\
\hline Palidez & 35 & $76.0 \%$ \\
\hline Fontanela anterior abo mbada & 33 & $71.7 \%$ \\
\hline Vómito & 28 & $60.8 \%$ \\
\hline Crisis convulsivas & 21 & $45.6 \%$ \\
\hline Sangrado de mucosas (paladar, conjuntivas) & 18 & $39.1 \%$ \\
\hline Historia de fiebre (no corroborada) & 16 & $34.7 \%$ \\
\hline Sangrado en heces & 14 & $30.4 \%$ \\
\hline Sangrado en sitios de punción & 14 & $30.4 \%$ \\
\hline Rechazo vía oral & 7 & $15.2 \%$ \\
\hline Distensión abdominal & 4 & $8.7 \%$ \\
\hline Hematuria & 4 & $8.7 \%$ \\
\hline Dificultad respiratoria & 1 & $2.17 \%$
\end{tabular}

( $n=46)$

Fuente: D epartamento de Informática y Estadística del Hospital del N iño Morelense 
La incidencia de la enfermedad hemorrágica tardía en países desarrollados es de 7 por cada 100000 nacimientos como máximo y la incidencia en países en desarrollo puede ser mayor. Los DALYs (disability-adjusted life-years) perdidos en niños menores de cinco años es de $0.1 \%$ a $0.2 \%$; traduciéndolo a costos de $\$ 1.00$ dólar por aplicación contra cada DALY salvado de $\$ 133.00$ dólares, ${ }^{2}$ lo cual representa claramente el beneficio de la prevención de esta entidad. El riesgo relativo para desarrollar EHRN entre los que reciben la profilaxis intramuscular con vitamina $\mathrm{K}$ y los que no la reciben es de 81:1. ${ }^{7}$ Asimismo, estudios epidemiológicos indican un pequeño pero significativo incremento en la incidencia de enfermedad hemorrágica después de la administración oral de vitamina K (1.0 a 6.4 casos por 100000 infantes), comparado con la administración intramuscular ( 0.25 casos por 100000 infantes), lo que apoya la indicación de esta última vía. ${ }^{8}$ Si se usa la vía oral, deberán repetirse la dosis en dos a tres ocasiones. ${ }^{2}$

La gran mayoría de los pacientes presentó la forma tardía $(91 \%)$ con sintomatología severa a nivel de sistema nervioso central. El 67\% presentó hemorragia intracraneana y $41 \%$ secuelas neurológicas severas; la frecuencia de muerte fue de $11 \%$, datos que coinciden con lo comunicado por Loughnan en cuyo estudio la edad media de presentación fue de 5.6 semanas, con hemorragias cerebrales en $63 \%$ de los casos, una tasa de mortalidad de $14 \%$ y secuelas cerebrales en $40 \%$ de los sobrevivientes. ${ }^{9}$

Llama la atención que en porcentajes muy similares se presentó atención del parto por médicos y parteras empíricas. Unicamente en 4\% se confirmó la aplicación de vitamina $\mathrm{K}$, y en la mayoría de los casos la familia ignoraba el dato. (Solves reportó enfermedad tardía aun con profilaxis intramuscular). ${ }^{5}$

El promedio de edad fue el esperado, considerando la presentación de tipo tardía, la cual ocurre después de dos semanas de vida, además de que todos tuvieron más de 38 semanas de gestación. ${ }^{8}$ Asimismo, la mayoría fue alimentada exclusivamente en el seno materno, lo cual se asocia con esta enfermedad debido a la baja concen- tración de vitamina $\mathrm{K}$ en la leche materna, y probablemente a la baja concentración de bacterias productoras de vitamina K2 (menaquinona) en el intestino. ${ }^{2}$

Es claro que debemos intensificar campañas para la promoción de la correcta atención del recién nacido en el posparto inmediato y los programas de capacitación sobre la prevención de EHRN al personal médico del primer nivel de atención, así como a las parteras empíricas, considerando que en nuestro estado del 25 al $35 \%$ de los partos son asistidos por estas últimas. Se deberá hacer una campaña dirigida a la población en general, principalmente a las mujeres en edad reproductiva, con el objetivo de que conozcan la necesidad de aplicación de la vitamina $\mathrm{K}$ en sus neonatos. Finalmente, las autoridades del sector salud deberán asegurar el abastecimiento y la aplicación de vitamina K, de acuerdo con la Norma Oficial Mexicana.

\section{Referencias}

1.Townsend CV.The hemorrhage diseases of the newborn. Arch Pediatr 1894;11:559.

2. Victora CG, Van Haecke P. Vitamin $\mathrm{K}$ prophylaxis in less developed countries: Policy issues and relevance to breastfeeding promotion. Am J Public Health 1988;88(2):203-209.

3. Segel George B. Hematology of the newborn. En: Beutler E, Lichtman MA, C oller BS, KippsTJ,W illiams Hematology, ed. Fifth edition. MacG rawHill. N ueva York, USA, 1995.

4.Zipursky A. Prevention of vitamin $\mathrm{K}$ deficiency bleeding in newborns. $\mathrm{Br}$ J Haematol 1999;101(3):130-137.

5. Solves P,A Ites A, G inovart G, Demestre J, Fontcuberta J. Late hemorrhage disease of the newborn as a cause of intracerebral bleeding.Ann Hematol 1997;75(1-2):65-66.

6. O rganización Panamericana de la Salud, (O PS). 0 rganización Mundial de la Salud, (O MS). C lasificación estadística internacional de enfermedades y problemas relacionados con la salud. Décima edición.W ashington, D.C.: O PS 1995, vol. 1.

7. MCN inch AW, Tripp JH. Haemorrhagic disease of the newborn in the British Isles:Two years prospective study. BMJ 1991;303(6810):1105-1109. 8. McMillan Douglas D. Administration of vitamin $\mathrm{K}$ to newborns: Implications and recommendations. CMAJ 1996;154:347-349.

9. Loughnan PM, MCD ougall PN . Epidemiology of late onset hemorrhage disease:A pooled data analysis. J Paediatr Child Health 1993;29:177-181. 